\title{
CASE OF STRICTURE OF SIGMOID FLEXURE OF THE COLON.
}

\author{
Br J. P. DOYLE, L.R.C.P.;
}

Surgeon to the Hospital for Children.

[Read in the Section of Medicine, March 11, 1892.

A LLARRIED man, forty-five year's of age, came under my care in October, 1889, complaining of soreness about the anus, tenesmus with passage of mucus, a feeling as if he were sitting on a plug, and pains in the abdomen and lumbar region. For a considerable time he had suffered from habitual coustipation, and for the previous six months the bowels did not act without purgatives, which were followed at times by a little diarrhoea. He had great trouble from the motions being very tenacious. There was tenderness on pressure over the left iliac and lumbar regions, and the abdomen was hard. Examination per anum caused great pain, the sphincters were very contracted, the rectum was empty but felt swollen, the heart and lungs were normal, pulse regular, 64 ; temp. $97^{\circ} \mathrm{Fahr}$. No albumen or tubecasts in urine. On November the 5 th, as he had not a motion for some days, and abdomen was distended and painful, I gave him an enema for the first time, but he could not retain more than six or eight ounces of the fluid, which was returned immediately along with some opaque gelatinous mucus. Pains in abdomen frequently set in after dinner, and on December 19 they were so severe that he had a weakness, attended with convulsive movements. Before the bowels acted he had griping and colicky pains, with a dull aching about the lower part of centre of lumbar 


\section{Case of Stricture of Sigmoid Flexure of the Colon.}

and sacral regions. After a motion, for about two hours, there were pains and soreness in the abdomen; at times he passed quantities of red or clear gelatinous mucus mixed with or adherent to the fæcal matter. On January 5, 1890, I could distinctly make out the descending colon and sigmoid flexure distended; they were very tender on pressure. Shortly after my examination he had two large evacuations, and on the following day all hardness and tenderness had disappeared. On March 27 I gave him at long enema tube and taught him its use. On April 3, after a walk, he got an attack of diarrhoea; and again, on May 13, he felt very ill, his bowels being very free. The integument of the lower part of the left side of his abdomen had become, from constant hot applications, of a dark mottled brown colour. He had been suffering very much from flatulence, and found from experience that milk diet suited him best. Between Oct. 15, 1890, and April 16,1891 , he progressed favourably, all his symptoms being very much improved, and he was able to pass the enema tube with greater ease. On April 18 he had an attack of obstruction with peritonitis; and again, on the 2nd of July, he had a similar attack, both produced by indiscretions in his diet. On July 8 passed some blood and mucus after using enema. August 26, while on the commode after an enema, he felt a sudden pain in the anus, which extended upwards, and gradually over abdomen. When I saw him on the following day he had all the symptoms of ruptured intestine with obstruction, which was followed by peritonitis, of which he died on September 4 .

This patient received great relief from opiates, baths, enemata of warm water, mixed at times with antiseptics; he frequently used 4 or 5 injections daily. Large doses of pulv. ipecac. were also of service. The character of the abdominal pains varied, being described as continuous, 
colicky, or griping, and frequently there was a sensation as if a tight girdle was round epigastrium.

Post mortem. - The abdominal cavity contained some reddish-brown fluid, the peritoneum was inflamed, fresh lymph was thrown out over the intestines; the rectum, descending colon, and sigmoid flexure were bound down by old adhesions and agglutinated by recent ones to neighbouring portions of the viscera; parts of the colon and small intestine had an ecchymosed appearance. The rectum was empty; the mucous membrane, of which the greater portion looks very thin and smooth, and nearly devoid of rugæ, presenting the appearance very much like that described as being present in so-called ballooning of the rectum. The ileum, ascending, transverse, and descending colon contained fæces; the latter was distended and packed with them, as far as the stricture in the sigmoid flexure. The rupture had taken place at the position of the kink at the inner side of the intestine, and apparently below the narrowing, as the opening in the stricture, which would admit about a No. 10 catheter, could be seen, and bulging against it but not escaping into the peritoneal cavity, was a hardened mass of fæces; part of the strictured tissues were included in the rent. The outer part of the strictured. tissues consists of a narrow hard band, and looks as if produced by the contraction of the cicatrix of an ulcer. The walls of the colon, for 8 or 10 inches above stricture, are in parts very much thickened, of a dark purplish-red colour, and the lumen is diminished. Signs of inflammatory changes are seen here and there as far up as the ileum.

The immediate cause of the rupture in this case is, I think, not common; it was produced by the over-distension of the intestine below the seat of narrowing with water after an enema used by the patient, as after injecting the bowel, and while sitting and straining on the stool the 


\section{Case of Stricture of Sigmoid. Flexure of the Colon.}

rupture occurred. The rent in the intestine, when seen on opening the abdominal cavity, was principally below the stricture, and looked as if the tissues, which had filled up the kink in the sigmoid flexure, had been torn through. The hard fæcal mass was pressed against the small opening in the stricture, but none escaped into the peritoneal cavity. The diagnosis of the conditions that existed in this cas. when it first came under my observation was by no means easy, and I could only treat symptoms until the nature of the lesions gradually evolved. The microscopical examination of the gelatinous mucus was of use in giving no evidence of a malignant nature. I found the examination of the rectum and colon by the long tube, while administering an enema, assist in determining the existence of stricture. 